\title{
Optimization of Automotive Suspension System by Design of Experiments: A Nonderivative Method
}

\author{
Anirban C. Mitra, Tanushri Soni, and G. R. Kiranchand \\ Department of Mechanical Engineering, MES's College of Engineering, Wadia College Campus, 19 Late Prin, \\ V. K. Joag Path, Pune 411001, India \\ Correspondence should be addressed to Anirban C. Mitra; bile007m@yahoo.com
}

Received 20 April 2016; Accepted 22 June 2016

Academic Editor: Marek Pawelczyk

Copyright (C) 2016 Anirban C. Mitra et al. This is an open access article distributed under the Creative Commons Attribution License, which permits unrestricted use, distribution, and reproduction in any medium, provided the original work is properly cited.

\begin{abstract}
A lot of health issues like low back pain, digestive disorders, and musculoskeletal disorders are caused as a result of the whole body vibrations induced by automobiles. This paper is concerned with the enhancement and optimization of suspension performance by using factorial methods of Design of Experiments, a nonderivative method. It focuses on the optimization of ride comfort and determining the parameters which affect the suspension behavior significantly as per the guidelines stated in ISO 2631-1:1997 standards. A quarter car test rig integrated with a LabVIEW based data acquisition system was developed to understand the real time behavior of a vehicle. In the pilot experiment, only three primary suspension parameters, that is, spring-stiffness, damping, and sprung mass, were considered and the full factorial method was implemented for the purpose of optimization. But the regression analysis of the data obtained rendered a very low goodness of fit which indicated that other parameters are likely to influence the response. Subsequently, steering geometry angles, camber and toe and tire pressure, were included in the design. Fractional factorial method with six factors was implemented to optimize ride comfort. The resultant optimum combination was then verified on the test rig with high correlation.
\end{abstract}

\section{Introduction}

Automobiles travel at a high speed and as a consequence experience a broad spectrum of random noncyclic vibrations transmitted either by tactile, visual, or aural paths. The term "ride" is commonly used to represent tactile and visual vibrations, while the audibly perceptible vibrations are categorized as "noise." As per SAE J670e [1] terminology, the term ride is defined as the low frequency vibrations of the sprung mass up to $5 \mathrm{~Hz}$. The lower frequency ride vibrations are manifestations of dynamic behavior. The motions and mechanical stresses resulting from the application of mechanical forces to the human body can have a variety of physiobiological effects. ISO 2631-1:1997 [2] standards imply that, for vertical vibrations, humans are most sensitive to those in the frequency range of 4 to $8 \mathrm{~Hz}$, this being the resonant frequency range for human body. In its simplest form, a modern road vehicle suspension has been defined by Damian [3] as a linkage to allow the wheel to have relative motion with respect to the body and to support loads while allowing for that motion.

The RC has been shown to be affected by a variety of suspension parameters and a variety of analytical, numeric, computational, and experimental methods have been exercised for the optimization of suspension systems. Khajavi et al. [4] have developed a full car 8-DOF model by adopting ride comfort, handling, and suspension travel as the main criteria and using Multiobjective Programming Discipline to find the Pareto front. A 33-DOF multibody model of suspension system has been prepared by Zhang et al. [5] using ADAMS software. Even the stiffness coefficient of bushing has been considered. Step and sinusoidal excitations are provided to the model, and RC is optimized using GA in one of the research works by Farid et al. [6].

Kilian et al. [7] had worked on the optimization of torsion, bending, and swaying of suspension designs by using finite element methods like topology optimization and topography optimization in Altair Opti Struct software to maximize ride 
comfort during the design stage. In a similar work, a twodimensional 8-DOF model was developed by Roy and Liu [8] to simulate and animate the response of a vehicle to different road, traction, braking, and wind conditions in a $3 \mathrm{D}$ VRML environment. A model validation was conducted by comparing a $0-100 \mathrm{kmph}$ acceleration run against a Honda Accord car equipped with an accelerometer and an engine rpm recorder. Gonçalves and Ambrósio [9] have proposed a methodology for optimization of ride and stability of a vehicle based on the use of flexible multibody model. The ride optimization is achieved by finding the optimum of ride index by measuring acceleration in several key points of the vehicle. Time histories of acceleration, velocities, and displacement at the center of gravity have been considered by Naudé and Snyman $[10,11]$ along with the time histories of forces, deflections, and deflection rate of wheels and suspension components. The presence of noise discontinuities presents major challenges in optimization. Multiobjective Genetic Algorithm (MOGA) is used for Pareto optimization of a 4 -DOF vehicle vibration model by Sharifi and Shahriari [12].

Bagheri et al. [13] has used GA for Pareto optimization of a 2-DOF vehicle vibration model considering sprung mass acceleration and relative displacement between sprung mass and tire. Evaluation of vibration transmitted from the road profile to the driver or a passenger in a moving vehicle has been done by Kuznetsov et al. [14]. The paper by Chi et al. [15] presents a comparative study of three optimization algorithms, namely, Genetic Algorithms (GAs), Pattern Search Algorithm (PSA), and Sequential Quadratic Program (SQP), for the design optimization of vehicle suspensions based on a quarter-vehicle model. Uys et al. [16] generated a Land Rover Defender model in MSC ADAMS and the spring damper settings were determined which ensures optimal ride comfort of an off-road vehicle at different speeds and over different road profiles.

A 7-DOF full car model has been developed and optimum ride comfort has been achieved by trying out different spring damper setting using DoE by Mostaani et al. [17]. Road surface has been simulated using power spectral density (PSD) and it was found that car spring stiffness is most sensitive. RMS acceleration and pitch angle for optimum setting at different speeds was generated and only ride comfort has been optimized. Marzbanrad et al. [18] performed optimization of passive suspension system on a 7-DOF model in MATLAB using DoE for speeds ranging from $60 \mathrm{kmph}$ to $90 \mathrm{kmph}$.

In this work, a quarter car test rig has been developed so as to vary the influential parameters within the predetermined sampling range. The test rig has been integrated with NI LabVIEW DAQ system for evaluating and assimilating the raw data. Subsequently, the DoE methodology has been implemented to optimize the suspension system. This paper has been organised in the following manner: a brief description of recent development in the field of suspension performance, RC, and Optimization processes is given in Section 1. Section 2 describes the experimental setup and measurement technique used for the accumulation of raw data. Section 3 focuses on the optimization process

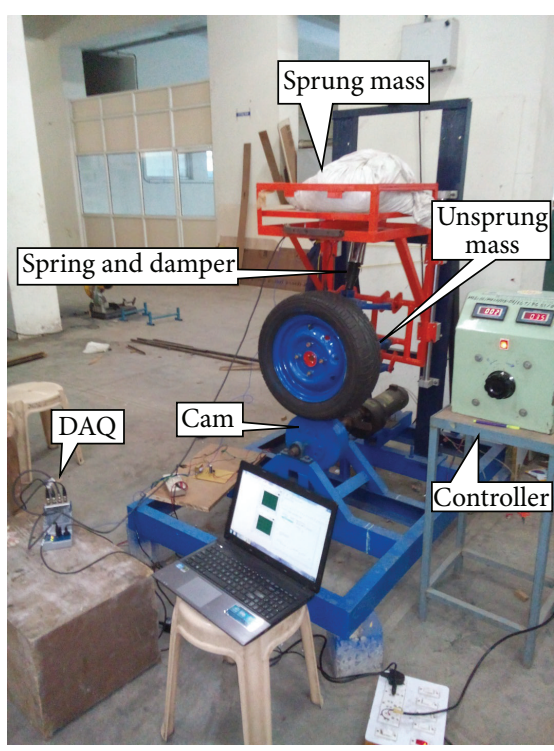

FIGURE 1: Quarter car test rig.

using DoE. Section 3.1 comprises the optimization using full factorial method; in which three fundamental suspension parameters, namely, the sprung mass $(m)$, spring stiffness (ks), and damping coefficient (cs), are considered. The section also elaborates various aspects of the regression analysis used in optimization process. The regression analysis indicates the presence of additional influential factors which have been left uncontrolled. Hence, in Section 3.2, three more parameters were added and the fractional factorial methodology is used for optimizing RC, hence considering six parameters, that is, the sprung mass $(m)$, spring stiffness $(\mathrm{ks})$, damping coefficient (cs), camber angle (cma), and toe and tire pressure (typ). Then, Section 4 explains the method of response optimization and the model generated. The verification of results and the concluding remarks have been mentioned in Sections 5 and 6, respectively.

\section{Quarter Car Suspension Test Rig}

In order to study the suspension behavior in controlled environment and to evaluate the influence of various factors and their interactions over RC, a quarter car suspension test rig was designed and developed as shown in Figure 1. The excitation source is a sinusoidal bump profile and the wheel of quarter car model is considered as follower. The cam profile has been actuated with a motor so as to simulate the relative motion between the road and the wheel. A provision to vary a multitude of parameters within the predetermined sampling range has been incorporated.

Two highly sensitive accelerometers, of ICP (IEPE) make (model: 351B03), have been attached to sprung mass and the wheel assembly to measure the acceleration of the sprung mass and the unsprung mass, respectively, and, as per ISO 2631-1:1997 [2] standards, RC is expressed in terms of RMS acceleration of the sprung mass in $\mathrm{m} / \mathrm{s}^{2}$. 


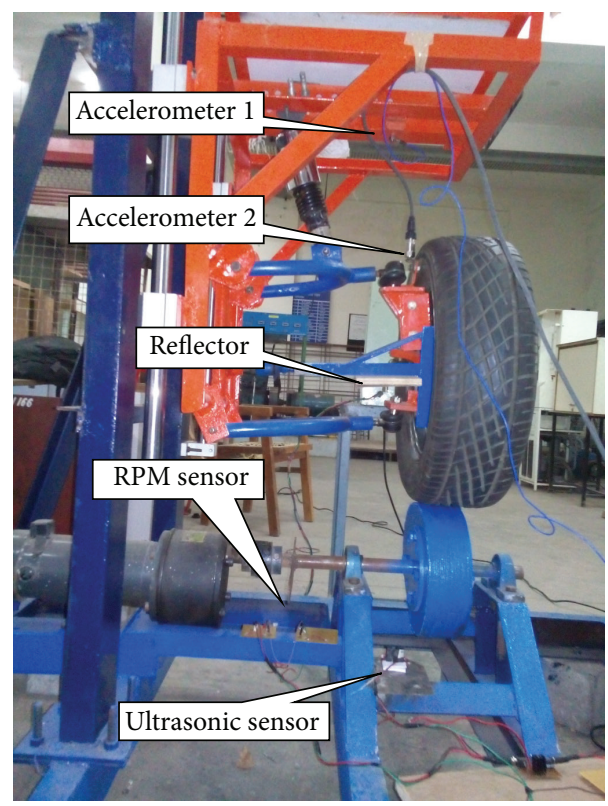

FIGURE 2: Sensors mounting.

The entire system has been integrated with NI LabVIEW data acquisition system, a development environment for a visual programming language from National Instruments. The mounting of accelerometer on control arms has been done as explained by Mohan et al. [19]. Arraigada and Partl [20] have discussed problems involving theoretical and analytical aspects of sensor calibration, data collection, and error identification and, as per authors' observations, the entire system has been integrated with NI LabVIEW data acquisition system and positions of sensors are shown in Figure 2.

\section{Design of Experiments}

DoE is a statistical technique used in this work to determine the various influential parameters affecting the response, that is, RC and the settings of those parameters in order to optimize the performance. The empirical relationship between the factors has also been derived in the form of equation by using the concept of regression analysis, which describes the relation between the factors and responses statistically. DoE quantifies the effects, variations, and uncertainty in the process and normalizes the data on a common scale in order to assist the analysis.

\subsection{Full Factorial DoE Analysis with Three Parameters. In} this section, the three fundamental variables sprung mass, spring stiffness, and the damping coefficient were considered to optimize the suspension system to enhance the RC of the vehicle. For the purpose of sampling range determination, an SAE BAJA buggy was taken as the reference. Accordingly, the softest and the hardest of springs and dampers as available in the Indian market were taken to be the extremities of the sampling range and the one-fourth of the total mass of a buggy was considered and the sprung mass was decided to be
TABLE 1: Full factorial design.

\begin{tabular}{lc}
\hline Design parameter & Value \\
\hline Factors & 3 \\
Base design & 3,8 \\
Runs & 16 \\
Replicates & 2 \\
Blocks & 2 \\
\hline
\end{tabular}

TABLE 2: Levels of influential parameters in full factorial design.

\begin{tabular}{lcc}
\hline Factors & Low $(-1)$ & High $(+1)$ \\
\hline Spring stiffness $(\mathrm{ks}), \mathrm{N} / \mathrm{mm}$ & 18000 & 26000 \\
Damping coefficient $(\mathrm{cs}), \mathrm{N}-\mathrm{s} / \mathrm{m}$ & 418 & 673 \\
Mass $(m), \mathrm{kg}$ & 41 & 81 \\
Speed $(N), \mathrm{RPM}$ & 150 & 250 \\
\hline
\end{tabular}

varied between $40 \mathrm{~kg}$ and $80 \mathrm{~kg}$ in the test rig. The speed of the vehicle here determines the frequency with which excitations are encountered by the vehicle body. While designing this experiment, the speed of the vehicle was not considered as a controlled variable because it depends completely on the user's aspirations. But, due to its considerable impact on the response, that is, RC, it was necessary to arrange for the elimination of variability due to its effect. So, the rotational speed of wheel in rpm was incorporated within two blocks. Mohan et al. [19] have surmised that bump traversal for a car can occur within a speed range of $10 \mathrm{kmph}$ to $20 \mathrm{kmph}$. Consequently, it was decided to vary the rotary speed of the cam from $150 \mathrm{rpm}$ to $250 \mathrm{rpm}$. The damping of the bearings, tires, and the body are small enough not to be considered.

Since only three variables have been considered for optimization, the full factorial approach was implemented. In this approach, no aliasing or compounding effect occur between the parameters. For two levels of each factor, the design is denoted as $2^{k}$ full factorial design where $k$ is the number of factors in the study. This method accounts for the effect of all the interactions and for reducing variability in the design; all the runs were replicated twice hence resulting in 16 runs. According to Montgomery [21], an obvious risk when conducting an experiment that has only one run at each test combination is that the model is fitted to noise and, with only one replicate in the design, the pure error and lack of fit cannot be estimated. The experiment was performed by considering two levels for each factor along with two blocks for speed. In a replicated blocked design, each replicate of the design is considered within a block and the design features have been depicted in Tables 1 and 2.

The experimentation was performed as per the orthogonal design matrix generated in MINITAB and the corresponding $\mathrm{RC}$ values were tabulated as shown in Table 3.

As evident from Table 3, the RMS values of sprung mass acceleration vary from $0.285 \mathrm{~m} / \mathrm{s}^{2}$ to $1.38 \mathrm{~m} / \mathrm{s}^{2}$. ISO 2631 1:1997 [2] clearly specifies that the sprung mass acceleration above $0.315 \mathrm{~m} / \mathrm{s}^{2}$ is a little uncomfortable whereas that above $0.5 \mathrm{~m} / \mathrm{s}^{2}$ is fairly uncomfortable for a passenger. Hence, the 
TABLE 3: Observation table: full factorial design.

\begin{tabular}{lccccc}
\hline $\begin{array}{l}\text { Run } \\
\text { order }\end{array}$ & Speed & $m$ & $\mathrm{ks}$ & $\mathrm{cs}$ & $\mathrm{RC}$ \\
\hline 1 & 150 & 41 & 26000 & 418 & 0.35 \\
2 & 150 & 81 & 26000 & 418 & 0.285 \\
3 & 150 & 81 & 26000 & 673 & 0.87 \\
4 & 150 & 81 & 18000 & 418 & 0.34 \\
5 & 150 & 41 & 18000 & 673 & 0.535 \\
6 & 150 & 81 & 18000 & 673 & 1.38 \\
7 & 150 & 41 & 26000 & 673 & 0.71 \\
8 & 150 & 41 & 18000 & 418 & 0.41 \\
9 & 250 & 81 & 26000 & 418 & 0.565 \\
10 & 250 & 41 & 26000 & 673 & 0.99 \\
11 & 250 & 81 & 18000 & 673 & 1.1 \\
12 & 250 & 41 & 18000 & 418 & 0.66 \\
13 & 250 & 41 & 26000 & 418 & 0.47 \\
14 & 250 & 81 & 18000 & 418 & 0.665 \\
15 & 250 & 81 & 26000 & 673 & 0.74 \\
16 & 250 & 41 & 18000 & 673 & 0.72 \\
\hline
\end{tabular}

main aim of this work is to obtain the optimal combination of suspension parameters so as to restrict the magnitude of the sprung mass acceleration below $0.315 \mathrm{~m} / \mathrm{s}^{2}$.

3.1.1. Regression Analysis. Regression analysis is the science of fitting straight lines to patterns of data. In a linear regression model, the dependent variable (RC in this case) is predicted from " $n$ " independent variables (here ks, cs, and $m$ ) using a linear equation and residuals are calculated in order to get the estimates of errors in model.

Before proceeding for the analysis, firstly the model is reduced and the unnecessary and insignificant parameters present in the model are removed. The presence of insignificant parameters inflates the biased error and decreases the model accuracy and adequacy. The estimated coefficient tables and the Pareto charts have been generated for this purpose.

The coefficient table gives the quantitative effect of each parameter on the response which is calculated by considering the average effects of the rest of the parameters. Table 4 gives the effect value of damping coefficient as 0.4125 , which shows that it has the highest effect on RC. Here, stiffness and sprung mass are individually insignificant for $\mathrm{RC}$ as their respective $P$ values are 0.190 and 0.094 , which is more than 0.05 . Also, a Pareto chart of the effects used to determine the magnitude and the importance of an effect visually is shown in Figure 3. There is a reference line on the chart which corresponds to the critical $t$ value $(t=2.306)$ and any effect that exceeds this reference line is significant.

Figure 3 shows that the damping coefficient and the interaction of the three variables are significant; hence the parameters like mass and stiffness cannot be neglected even after being individually insignificant.
TABLE 4: Effect coefficient table: full factorial design.

\begin{tabular}{lccccc}
\hline Term & Effect & Coef & SE Coef & $t$ & $P$ \\
\hline Constant & 0.67438 & 0.03624 & 18.61 & 0.000 & \\
Block & -0.06437 & 0.03624 & -1.78 & 0.114 & \\
ks & -0.10375 & -0.05188 & 0.03624 & -1.43 & 0.190 \\
cs & 0.41250 & 0.20625 & 0.03624 & 5.69 & 0.000 \\
$m$ & 0.13750 & 0.06875 & 0.03624 & 1.90 & 0.094 \\
ks $*$ cs & -0.15250 & -0.07625 & 0.03624 & -2.10 & 0.069 \\
cs $* m$ & 0.14625 & 0.07313 & 0.03624 & 2.02 & 0.078 \\
ks $*$ cs $* m$ & -0.17625 & -0.08813 & 0.03624 & -2.43 & 0.041 \\
\hline
\end{tabular}

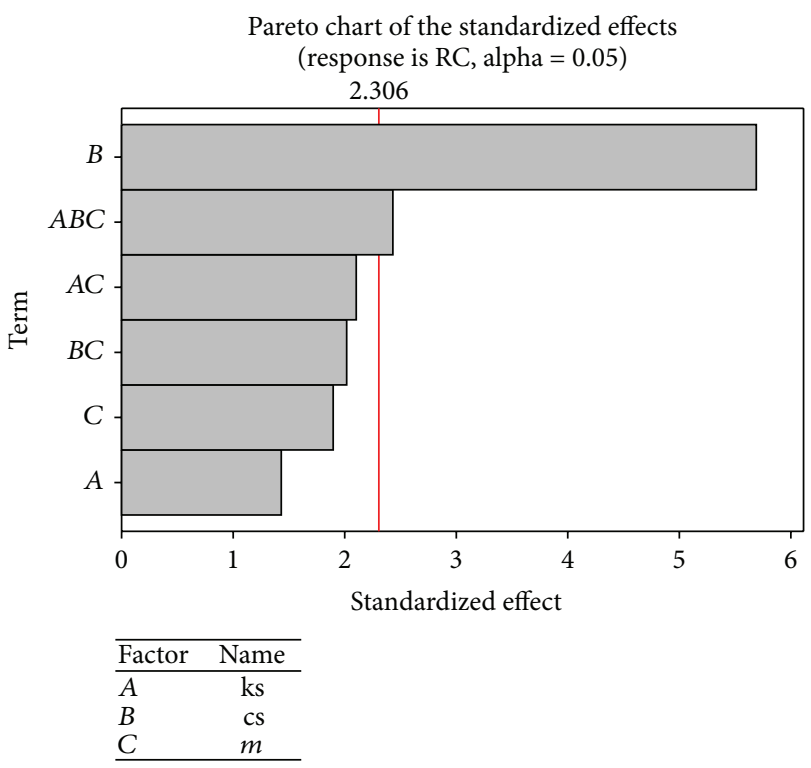

Figure 3: Pareto chart: full factorial design.

3.1.2. Goodness of Fit. Now, the model of RC can be analyzed to check its adequacy and fitment. Three statistics are used in Ordinary Least Squares regression to evaluate model fit: $R$-Squared, standard error $(S)$, and the overall $F$-test. Hence, the statistical terms such as standard error, coefficient of determination, and predictability values were calculated for the response as shown in Table 5.

$S$ stands for standard error and is 0.145 for the model. It is an estimate of the standard deviation of the true noise; that is, the variations in the response that are not explained by the model. The smaller the value of $S$, the greater the closeness of experimental responses with the fitted line and the accuracy of the model. The $R$-Squared value (coefficient of determination) is $87.42 \%$, which gives an estimate of explained variation in the model and determines how closely a certain function fits a particular set of experimental data. $R$ Squared adjusted value, $76.41 \%$, is assessed. The perceptible difference between the values of $R$-Squared and $R$-Squared adjusted here shows that some influential parameters might not have been considered for this analysis.

Along with this, the PRESS value in the model is 0.672 , which is comparatively larger than the ideal value. Similarly, 
TABLE 5: Regression statistics: full factorial design.

\begin{tabular}{lcc}
\hline Term & & Value \\
\hline$S$ & Standard error & 0.145 \\
PRESS & Prediction sum of squares & 0.672 \\
$R$-Squared & Coeff of multiple determination & $87.42 \%$ \\
$\begin{array}{l}R \text {-Squared } \\
\text { (pred) }\end{array}$ & Predicted coeff of determination & $49.68 \%$ \\
$\begin{array}{l}R \text {-Squared } \\
\text { (adj) }\end{array}$ & Adjusted coeff of determination & $76.41 \%$ \\
\hline
\end{tabular}

the $R$-Squared predicted value is $49.68 \%$ which depicts a very low predictability and that the model is unable to account for the variability in the model. Hence, it was surmised that, apart from the primary parameters, other factors are likely to influence the response and can be included in the design. Consequently, it was decided to take steering geometry parameters into consideration along with the tire pressure and obtain a setting of all the parameters in order to minimize the RC.

3.2. Fractional Factorial DoE Analysis with Six Parameters. This section deals with identifying the other influential parameters and then optimizing the RC using fractional factorial method. The process comprises extensive literature survey and study of vehicle dynamics. Study of steering geometry reveals that the alignment and orientation of the wheels and the suspension linkages also play a nodal role in altering the area of contact at the tire-road interface. One such parameter is tire camber which determines the orientation along which the normal reaction generated at the tire-road interface is transferred to the vehicle body. Another aspect of the steering geometry, namely, the toe, is also said to have a profound impact on the directional stability and the self-straightening ability of the general passenger car. It has been mentioned in SAE J670e [1] that the toe determines the magnitude and orientation of lateral forces acting on the wheel assembly under dynamic conditions.

The generation of the forces necessary to initiate the turn, to constrain the vehicle at the correct sideslip angle, and to return it to the straight-running condition is the role of the tires. Moreover, as the tire is made of rubber, which is partially elastic, the tire is analogous to a spring. Pillai [22] has stated that the stiffness of the tire is likely to affect the nature of the vibrations transmitted to the vehicle and as the tire stiffness is said to be a direct function of tire pressure, it was decided to incorporate camber angle, toe, and tire inflation pressure among the parameters.

3.2.1. Fractional Factorial Design. As the number of parameters increases to 6 , in the subsequent section of this paper, fractional factorial methodology has been implemented to create and analyze the design by considering the 2 levels of six influential parameters. Fractional factorial design is an efficient alternative for full factorial design.

Here, the fractional factorial design is selected by defining the design generator such that it minimizes the aliasing effect
TABLE 6: Fractional factorial design.

\begin{tabular}{lc}
\hline Design parameter & Value \\
\hline Factors & 6 \\
Base design & 6,16 \\
Runs & 32 \\
Replicates & 2 \\
Blocks & 2 \\
Resolution & IV \\
\hline
\end{tabular}

TABLE 7: Levels of influential parameters in fractional factorial design.

\begin{tabular}{lcc}
\hline Factors & Low $(-1)$ & High $(+1)$ \\
\hline Speed $(N)$, RPM & 150 & 250 \\
Tire pressure (typ), psi & 35 & 40 \\
Camber (cma), degree & 1 & 3 \\
Spring stiffness (ks), N/mm & 18000 & 26000 \\
Damping coefficient (cs), N-s/m & 418 & 673 \\
toe, mm & 10 & 20 \\
Mass $(m), \mathrm{kg}$ & 41 & 81 \\
\hline
\end{tabular}

in the design depending upon the resolution chosen. Here, to obtain a $2^{6-2}$ fractional factorial design, (1/4)th fraction of $2^{6}$ design requires 2 design generators to be defined which are $E=A B C$ and $F=B C D$. Now, those runs are selected which give same sign for the generators and the identity column. Hence, the defining relation, which is the total collection of all the design generators, can be given as

$$
I+A B C E+A D E F+B C D F,
$$

where $A, B, C, D, E$, and $F$ are parameters and $I$ indicates the identity column.

Based on the defining relation, the alias structure is obtained which describes the confounded effects in a design. Aliasing or confounding in a design occurs when the estimate of an effect includes the influence of one or more other effects. The alias structures of this design show that all the main effects are confounded with third- and fifth-order effects but no main effect is aliased with any other main effect. The second-order effects are confounded with second- as well as fourth- and sixth-order effect and all the third order effects are confounded with each other. Consequently, the Resolution IV design was chosen as it does not confound the main effects with the two factor interactions.

Table 6 shows the overall summary of the fractional factorial design obtained as per the above discussion and the two levels of all the 6 factors have been shown in Table 7.

A Randomized Complete Block Design was formulated with two replicates and blocks, and the experimentation was performed accordingly as shown in Table 8.

3.2.2. Regression Analysis. To evaluate the nature of influence of each parameter over the response, the effects coefficient table was generated as shown in Table 9 . 
TABLE 8: DoE matrix: fractional factorial design.

\begin{tabular}{lcccccccc}
\hline Run & $N$ & typ & cma & ks & cs & toe & $m$ & RC \\
\hline 1 & 155 & 35 & 3 & 18000 & 418 & 10 & 41 & 0.48 \\
2 & 155 & 35 & 3 & 26000 & 673 & 20 & 81 & 0.67 \\
3 & 155 & 35 & 1 & 26000 & 673 & 10 & 41 & 0.8 \\
4 & 155 & 35 & 1 & 26000 & 673 & 10 & 41 & 0.72 \\
5 & 155 & 35 & 1 & 18000 & 418 & 20 & 81 & 1.24 \\
6 & 155 & 35 & 3 & 18000 & 418 & 10 & 41 & 0.45 \\
7 & 155 & 35 & 3 & 26000 & 673 & 20 & 81 & 0.65 \\
8 & 155 & 35 & 1 & 18000 & 418 & 20 & 81 & 1.3 \\
9 & 250 & 35 & 3 & 18000 & 673 & 10 & 81 & 0.54 \\
10 & 250 & 35 & 3 & 26000 & 418 & 20 & 41 & 1.2 \\
11 & 250 & 35 & 3 & 26000 & 418 & 20 & 41 & 1.06 \\
12 & 250 & 35 & 1 & 26000 & 418 & 10 & 81 & 1.7 \\
13 & 250 & 35 & 1 & 26000 & 418 & 10 & 81 & 1.5 \\
14 & 250 & 35 & 1 & 18000 & 673 & 20 & 41 & 1.15 \\
15 & 250 & 35 & 1 & 18000 & 673 & 20 & 41 & 1.2 \\
16 & 250 & 35 & 3 & 18000 & 673 & 10 & 81 & 0.62 \\
17 & 155 & 40 & 3 & 26000 & 418 & 10 & 81 & 1.12 \\
18 & 155 & 40 & 1 & 18000 & 673 & 10 & 81 & 0.55 \\
19 & 155 & 40 & 1 & 26000 & 418 & 20 & 41 & 0.9 \\
20 & 155 & 40 & 3 & 18000 & 673 & 20 & 41 & 0.54 \\
21 & 155 & 40 & 1 & 26000 & 418 & 20 & 41 & 0.82 \\
22 & 155 & 40 & 1 & 18000 & 673 & 10 & 81 & 0.57 \\
23 & 155 & 40 & 3 & 26000 & 418 & 10 & 81 & 1.17 \\
24 & 155 & 40 & 3 & 18000 & 673 & 20 & 41 & 0.44 \\
25 & 250 & 40 & 1 & 18000 & 418 & 10 & 41 & 0.97 \\
26 & 250 & 40 & 1 & 26000 & 673 & 20 & 81 & 1.6 \\
27 & 250 & 40 & 3 & 26000 & 673 & 10 & 41 & 0.61 \\
28 & 250 & 40 & 3 & 18000 & 418 & 20 & 81 & 1.75 \\
29 & 250 & 40 & 1 & 18000 & 418 & 10 & 41 & 0.91 \\
30 & 250 & 40 & 3 & 18000 & 418 & 20 & 81 & 1.75 \\
31 & 250 & 40 & 1 & 26000 & 673 & 20 & 81 & 1.25 \\
32 & 250 & 40 & 3 & 26000 & 673 & 10 & 41 & 0.69 \\
\hline & & & & & & & &
\end{tabular}

TABLE 9: Effects and coefficients for RC: fractional factorial.

\begin{tabular}{lccccc}
\hline Term & Effect & Coef & SE Coef & $t$ & $P$ \\
\hline Const & & 0.9663 & 0.01647 & 58.67 & 0 \\
Blocks & & -0.19 & 0.01647 & -11.54 & 0 \\
typ (psi) & 0.0225 & 0.0113 & 0.01647 & 0.68 & 0.502 \\
cma (deg) & -0.215 & -0.1075 & 0.01647 & -6.53 & 0.029 \\
ks $(\mathrm{N} / \mathrm{m})$ & 0.125 & 0.0625 & 0.01647 & 3.79 & 0 \\
cs $(\mathrm{N}-\mathrm{s} / \mathrm{m})$ & -0.3575 & -0.1787 & 0.01647 & -10.85 & 0 \\
toe $(\mathrm{mm})$ & 0.2575 & 0.1288 & 0.01647 & 7.82 & 0 \\
$m$ & 0.315 & 0.1575 & 0.01647 & 9.56 & 0 \\
typ $(\mathrm{psi}) * \mathrm{cma}(\mathrm{deg})$ & 0.2775 & 0.1388 & 0.01647 & 8.42 & 0 \\
typ $(\mathrm{psi}) * m$ & 0.17 & 0.085 & 0.01647 & 5.16 & 0 \\
\hline
\end{tabular}

It can be observed from Table 9 that the SE Coef value of 0.01647 is much lower than that obtained when three parameters were considered. This is a direct indication that the
TABLE 10: Regression statistics: fractional factorial design.

\begin{tabular}{lcc}
\hline Term & & Value \\
\hline$S$ & Standard error & 0.093 \\
PRESS & Prediction sum of squares & 0.0403 \\
$R$-Squared & Coeff of multiple determination & $96.21 \%$ \\
$R$-Squared (pred) & Predicted coeff of determination & $91.98 \%$ \\
$R$-Squared (adj) & Adjusted coeff of determination & $94.66 \%$ \\
\hline
\end{tabular}

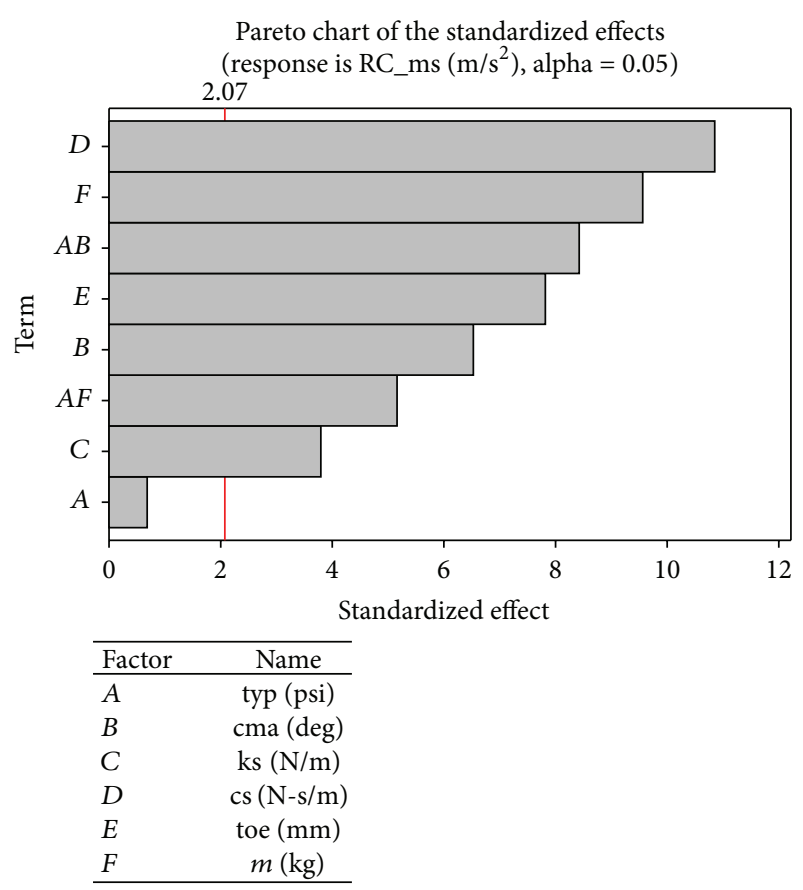

Figure 4: Pareto chart: fractional factorial design.

residual error in evaluating the system has been minimized after considering additional parameters. Moreover, all the individual parameters other than the tire pressure are found to be significant. In the previous instance, while taking three factors, the effect of the noise factors caused the individual influence of parameters like mass and spring stiffness to be clouded. This further emphasizes the importance of selection of influential variables in a DoE optimization procedure. A visual perception of the same can be derived from the Pareto charts which clearly demarcate the significant and insignificant influences, as shown in Figure 4.

The critical $t$ value is 2.07 . Tire pressure is insignificant individually but it cannot be neglected as its interactions are significant. The Pareto chart obtained also conforms to the Effect-Heredity principle, which states that, for an interaction to be significant, at least one of its parent factors should be significant. After reducing the model of RC to its significant variables, the model was checked for goodness of fit by evaluating the $R$ sq statistics shown in Table 10 .

The $S$ value obtained here is 0.093 which is relatively smaller and accounts for greater accuracy of the resulting model. The high value of $R$-Squared, that is, $96.21 \%$, and the closeness of $R$-Squared and adjusted $R$-Squared (94.66\%) 


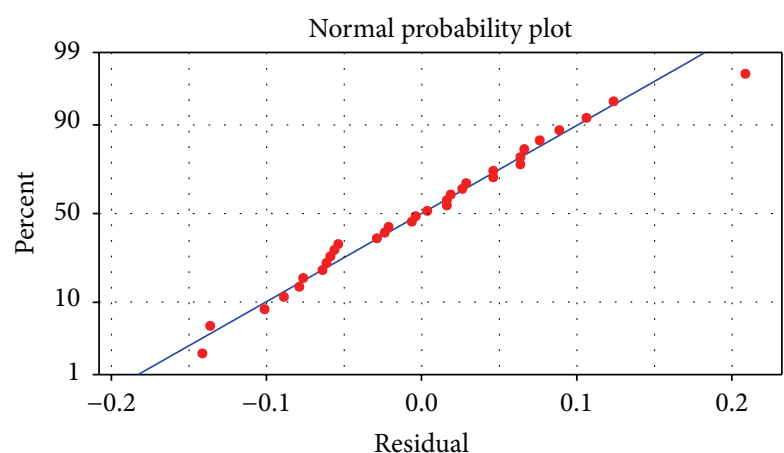

(a)

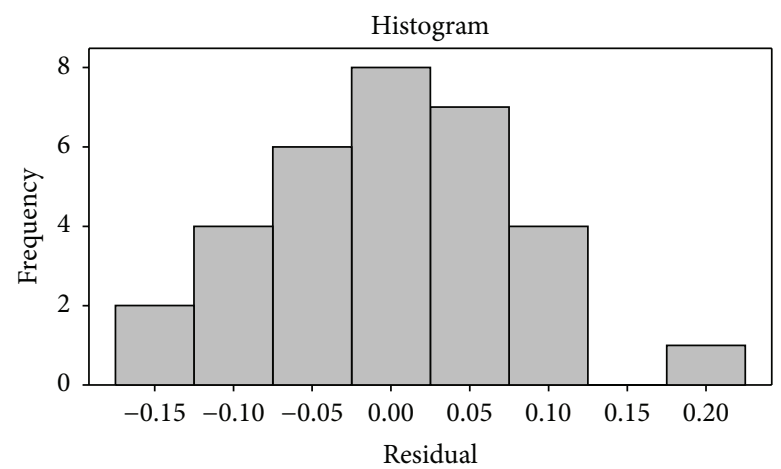

(c)

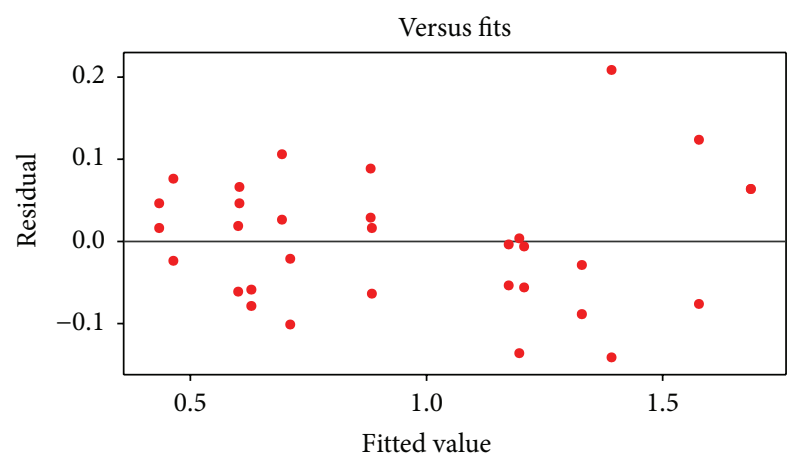

(b)

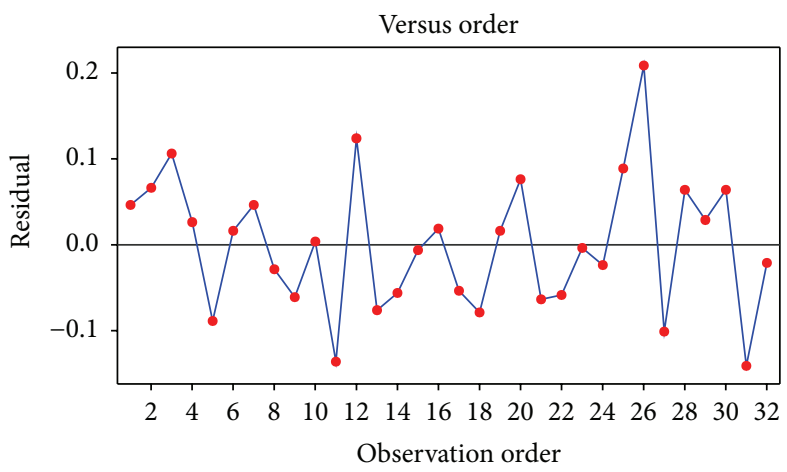

(d)

FIgURE 5: Residual plots for RC: fractional factorial design. Residual plots for RC_ms (m/s $\left.\mathrm{s}^{2}\right)$.

depicted in Table 10 confirm the fitment of model. PRESS and predicted $R$-squared values give an idea about the model's predictability and the value of PRESS here is smaller, that is, 0.0403 , compared to that when three factors were considered. Similarly, predicted $R$-Squared is $91.98 \%$. The higher value of predicted $R$-Squared shows better predictability and the negligible difference between adjusted $R$-Squared and $R$ Squared indicates that the model is not overfit.

The normal probability plot for RC, at Figure 5(a), indicates the presence of one outlier which can be neglected, as far as its impact on the model is concerned. In this analysis, the distribution of residuals over the zero line follows a bell shaped curve, but the presence of some outlier points on extreme left and right makes it slightly skewed. The versus-fit plots, at Figure 5(b), show a random and equal distribution without any usual patterns for both the responses which shows that the residuals possess a constant variance throughout the data. The graph at Figure 5(d) is the versus order plot which is the plot of residuals in time sequence. The plot is used to detect the correlation between the residuals. Ideally, the residuals on the plot should fall randomly around the center line. There is no strong pattern observed in the versus order plots for $\mathrm{RC}$ which indicate the random variation in experimental data. Hence, the residuals are deemed time independent and uncorrelated in the analysis.

3.2.3. ANOVA. ANOVA, that is, Analysis of Variance, explains the variability in the mean and variance of the residuals, taking into account the degree of freedom for each variable. The ANOVA table generated for RC is shown in Table 11. In ANOVA, the total sum of squares quantifies the total variation in the data and is divided into two parts, Seq SS (sequential sum of squares) and Adj SS (adjusted sum of squares). The equality of both these terms confirms the orthogonality of the design. The residual mean square (MS) of treatments is an unbiased estimator of variance.

Lower $P$ values provide stronger evidence against the null hypothesis; that is, for $P$ value less than 0.05 , the effect of the factor is termed significant and the null hypothesis can be rejected. As suggested by the $F$-value and $P$ value in Table 9 , rotational speed, which was considered within a block, is significantly influential.

The lack of fit for the model is also tested by using $F$-test. To obtain the lack of fit estimation, replication of the model is mandatory. There are two parts of the error term in the model, pure error and the bias error. The error due to variations in the replications around their mean value is called pure error; on the other hand bias error is due to the variation of mean values around the model prediction. Ideally, the $F$-ratio of lack of fit should not be significant for the model to describe the functional relationship between the experimental factors and the response. For the model, in Table 11, the $P$ value of lack of fit is 0.178 , which is greater than the significance level of 0.05 . This shows its insignificance which in turn depicts the fitment and credibility of the model generated. Also the variance of model error, that is, lack of fit and replicate error, that is, 
TABLE 11: ANOVA analysis: fractional factorial design.

\begin{tabular}{|c|c|c|c|c|c|c|}
\hline Source & $\mathrm{DF}$ & Seq SS & Adj SS & Adj MS & $F$ & $P$ \\
\hline Blocks & 1 & 1.1552 & 1.1552 & 1.1552 & 133.09 & 0 \\
\hline Main effects & 6 & 2.84555 & 2.84555 & 0.47426 & 54.64 & 0 \\
\hline typ (psi) & 1 & 0.00405 & 0.00405 & 0.00405 & 0.47 & 0.502 \\
\hline cma (degree) & 1 & 0.3698 & 0.3698 & 0.3698 & 42.61 & 0 \\
\hline $\mathrm{ks}(\mathrm{N} / \mathrm{m})$ & 1 & 0.125 & 0.125 & 0.125 & 14.4 & 0.001 \\
\hline cs $(\mathrm{N}-\mathrm{s} / \mathrm{m})$ & 1 & 1.02245 & 1.02245 & 1.02245 & 117.8 & 0 \\
\hline toe $(\mathrm{mm})$ & 1 & 0.53045 & 0.53045 & 0.53045 & 61.11 & 0 \\
\hline$m$ & 1 & 0.7938 & 0.7938 & 0.7938 & 91.46 & 0 \\
\hline Two-way interactions & 2 & 0.84725 & 0.84725 & 0.42363 & 48.81 & 0 \\
\hline typ (psi) * cma (deg) & 1 & 0.61605 & 0.61605 & 0.61605 & 70.98 & 0 \\
\hline typ $(\mathrm{psi}) * m$ & 1 & 0.2312 & 0.2312 & 0.2312 & 26.64 & 0 \\
\hline Residual error & 22 & 0.19095 & 0.19095 & 0.00868 & & \\
\hline Lack of fit & 6 & 0.07515 & 0.07515 & 0.01253 & 1.73 & 0.178 \\
\hline Pure error & 16 & 0.1158 & 0.1158 & 0.00724 & & \\
\hline Total & 31 & 5.03895 & & & & \\
\hline
\end{tabular}

pure error values for the RC model, is 0.01253 and 0.00724 , respectively, which was very negligible and it indicates that the experimentation is having good reproducibility.

3.2.4. Model with Six Variables. Once all the assumptions were verified and consolidated and the fit of model is obtained, an experimental model explaining the behavioral relationship between all the selected suspension and steering geometry parameters is shown in

$$
\begin{aligned}
\mathrm{RC}= & -0.210200 \times \text { typ }-2.18875 \times \mathrm{cma}+1.56250 E \\
& -05 \times \mathrm{ks}-0.00140196 \times \mathrm{cs}-0.0257500 \times \text { toe } \\
& -0.0558750 \times m+0.05550 \times \text { typ } \times \mathrm{cma} \\
& +0.001700 \times \text { typ } \times m-0.1900 \times N+8.61814 .
\end{aligned}
$$

\section{Response Optimization}

After the experimentation and the statistical analysis, the regression models for the desired responses are generated in terms of the influential parameters, which produce a value of response as per the given set of the factors. When the value of response is desired around a user-specified range and the combination of various influencing factors is required to yield the optimal response accordingly, the method of response optimization is applied. In this, the most desirable value of response possible under all the restrictions or conditions is achieved.

To serve the purpose, response optimizer, a software function in MINITAB, is used. It generates a combination of the variables which gives the optimal solution, based on the goal of optimization, range, weight, and relative importance as specified by the user. The model must fit all the responses separately, to be implemented in the response optimizer. The response can be minimized, maximized, or targeted as per the objective of optimization. RC is measured in terms of
RMS acceleration of sprung mass and improves as its value decreases; hence the response is to be minimized to obtain a high degree of comfort.

As recommended by ISO 2631-1:1997 [2] standards, RC should be less than $0.5 \mathrm{~m} / \mathrm{s}^{2}$, so the target value for RC was $0.3 \mathrm{~m} / \mathrm{s}^{2}$ and upper value was $0.6 \mathrm{~m} / \mathrm{s}^{2}$. An interactive optimizer plot in Figure 6 is also provided by the response optimizer which shows the optimum factor settings (values in red) and the response values (in blue) along with their respective desirabilities.

The predicted value(s) of response(s) can be calculated at a particular setting of interest for one or more factors and sensitivity of factors can be determined. When one or more input factors are changed to a new level, the graphs of desirabilities are redrawn and the predicted responses and desirabilities are recalculated. The model gives an optimum RMS acceleration of $0.2663 \mathrm{~m} / \mathrm{s}^{2}$ at the optimum combination of tire pressure of 35 psi, camber angle of 3 degree, toe of $10 \mathrm{~mm}$, spring stiffness of $18000 \mathrm{~N} / \mathrm{m}$, and damper of damping coefficient $673 \mathrm{~N}-\mathrm{s} / \mathrm{m}$ with mass of $41 \mathrm{~kg}$.

\section{Results and Verification}

The optimum setting for all the factors which minimizes the RMS acceleration, that is, improves the RC to the optimum level, is shown in Table 12. This optimum setting obtained is again verified by executing the combination on the test rig and the experimental value of RC was found to be $0.302 \mathrm{~m} / \mathrm{s}^{2}$ against the theoretical value of $0.2663 \mathrm{~m} / \mathrm{s}^{2}$.

\section{Conclusion}

In this work, the experimentation was accomplished by incorporating various combinations of the parameters on the test rig developed, for the simulation of real time behavior of suspension system of a vehicle. It can be concluded 


\begin{tabular}{|c|c|c|c|c|c|c|}
\hline $\begin{array}{cc}\text { Optimal } & \text { High } \\
D & \text { Cur } \\
1.0000 & \text { Low } \\
& \\
\end{array}$ & $\begin{array}{c}\text { typ (psi) } \\
40.0 \\
{[35.0]} \\
35.0 \\
\end{array}$ & $\begin{array}{c}\mathrm{cma}(\mathrm{deg}) \\
3.0 \\
{[3.0]} \\
1.0 \\
\end{array}$ & $\begin{array}{c}\mathrm{ks}(\mathrm{N} / \mathrm{m}) \\
26000.0 \\
{[18000.0]} \\
18000.0\end{array}$ & $\begin{array}{c}c \text { cs }(\mathrm{N}-\mathrm{s} / \mathrm{m}) \\
673.0 \\
{[673.0]} \\
418.0 \\
\end{array}$ & $\begin{array}{c}\text { toe }(\mathrm{mm}) \\
20.0 \\
{[10.0]} \\
10.0 \\
\end{array}$ & $\begin{array}{c}m \\
81.0 \\
{[41.0]} \\
41.0 \\
\end{array}$ \\
\hline \multicolumn{7}{|l|}{$\begin{array}{c}\text { Composite } \\
\text { desirability } \\
1.0000\end{array}$} \\
\hline $\begin{array}{c}\text { rms_accl } \\
\text { Minimum } \\
y=0.2663 \\
d=1.0000\end{array}$ & & & & & & \\
\hline
\end{tabular}

FIGURE 6: Response optimization plot.

TABLE 12: Results and verification.

\begin{tabular}{lc}
\hline Factors & Optimum level \\
\hline Tire pressure & $35 \mathrm{psi}$ \\
Camber & 3 degrees \\
Spring stiffness & $18000 \mathrm{~N} / \mathrm{m}$ \\
Damping coefficient & $673 \mathrm{Ns} / \mathrm{m}$ \\
toe & $10 \mathrm{~mm}$ \\
Mass & $41 \mathrm{~kg}$ \\
\hline Verification & \\
\hline RC (response optimization) & RC [experimental value (test rig)] \\
$0.2663 \mathrm{~m} / \mathrm{s}^{2}$ & $0.302 \mathrm{~m} / \mathrm{s}^{2}$ \\
\hline
\end{tabular}

from the aforementioned work that the RC, measured in terms of RMS acceleration of sprung mass in a vehicle, cannot be successfully optimized by controlling only spring stiffness, damping coefficient, and the sprung mass. The steering geometry angles, that is, camber and toe with the tire pressure, significantly affect the ride behavior of a vehicle along with the primary suspension parameters.

Also, it was found that the fractional factorial method of optimization reduces the number of runs significantly with the negligible compromise in the result, as the application of full factorial method is only limited to the experimentations where number of runs is small.

Later, the experimental model for RC and the optimized combination of six influential parameters were obtained after the regression analysis of data acquired, which was again verified on the test rig. As the responses have been treated with the road profile of a bump, it can be deemed that the optimized set of values will render a very comfortable ride as far as normal roads are considered.

\section{Competing Interests}

The authors declare that they have no competing interests.

\section{Acknowledgments}

The authors are thankful to the MESCOE-NI LabVIEW Academy Lab, Department of Mechanical Engineering,
MES's College of Engineering, Pune, India, for providing the necessary testing facilities.

\section{References}

[1] SAE J670e, Vehicle Dynamics Terminology. Standard, Society of Automotive Engineers, 1976.

[2] ISO, "Mechanical vibration and shock evaluation of human exposure to whole body vibration. Part 1: general requirements," ISO Standard 2631-1:1997, International Organization for Standardization, Geneva, Switzerland, 1997.

[3] H. Damian, Multibody Systems Approach to Vehicle Dynamics, Butterworth-Heinemann, Oxford, UK, 2004.

[4] M. N. Khajavi, B. Notghi, and G. Paygane, "Multi objective optimization approach to optimize vehicle ride and handling characteristics," World Academy of Science, Engineering and Technology, vol. 62, pp. 580-584, 2010.

[5] J. Zhang, Y. Zhang, and R. Gao, "Genetic algorithms for optimal design of vehicle suspensions," in Proceedings of the IEEE International Conference on Engineering of Intelligent Systems (ICEIS '06), pp. 1-6, IEEE, Islamabad, Pakistan, April 2006.

[6] T. M. Farid, A. Salah, and W. Abbas, "Design of optimal linear suspension for quarter car with human model using genetic algorithms," Journal of Applied Sciences Research, vol. 7, no. 11, pp. 1709-1720, 2011.

[7] S. Kilian, U. Zander, and F. E. Talke, "Suspension modeling and optimization using finite element analysis," Tribology International, vol. 36, no. 4-6, pp. 317-324, 2003.

[8] S. Roy and Z. Liu, "Road vehicle suspension and performance evaluation using a two-dimensional vehicle model," International Journal of Vehicle Systems Modelling and Testing, vol. 3, no. 1-2, pp. 68-93, 2008.

[9] J. P. C. Gonçalves and J. A. C. Ambrósio, "Road vehicle modeling requirements for optimization of ride and handling," Multibody System Dynamics, vol. 13, no. 1, pp. 3-23, 2005.

[10] A. F. Naudé and J. A. Snyman, "Optimisation of road vehicle passive suspension systems-part 1. Optimisation algorithm and vehicle model," Applied Mathematical Modelling, vol. 27, no. 4, pp. 249-261, 2003.

[11] A. F. Naudé and J. A. Snyman, "Optimisation of road vehicle passive suspension systems. Part 2. Qualification and case study," Applied Mathematical Modelling, vol. 27, no. 4, pp. $263-$ 274, 2003.

[12] M. Sharifi and B. Shahriari, "Pareto optimization of vehicle suspension vibration for a nonlinear halfcar model using a 
multi-objective genetic algorithm," Research Journal of Recent Sciences, vol. 1, no. 8, pp. 17-22, 2012.

[13] A. Bagheri, M. J. Mahmoodabadi, H. Rostami, and S. Kheybari, "Pareto optimization of a two-degree of freedom passive linear suspension using a new multiobjective genetic algorithm," International Journal of Engineering, Transactions A: Basics, vol. 24, no. 3, pp. 291-299, 2011.

[14] A. Kuznetsov, M. Mammadov, I. Sultan, and E. Hajilarov, "Optimization of a quarter-car suspension model coupled with the driver biomechanical effects," Journal of Sound and Vibration, vol. 330, no. 12, pp. 2937-2946, 2011.

[15] Z. Chi, Y. He, and G. F. Naterer, "Design optimization of vehicle suspensions with a quarter-vehicle model," CSME Transactions, vol. 32, no. 2, pp. 297-312, 2008.

[16] P. E. Uys, P. S. Els, and M. Thoresson, "Suspension settings for optimal ride comfort of off-road vehicles travelling on roads with different roughness and speeds," Journal of Terramechanics, vol. 44, no. 2, pp. 163-175, 2007.

[17] S. Mostaani, D. Singh, K. Firouzbakhsh, and M. T. Ahmadian, "Optimization of a passive vehicle suspension system for ride comfort enhancement with different speeds based on DOE method," in Proceedings of the International Joint Colloquiums on Computer Electronics Electrical Mechanical and Civil (CEMC '11), Kerala, India, September 2011.

[18] J. Marzbanrad, M. Mohammadi, and S. Mostaani, "Optimization of a passive vehicle suspension system for ride comfort enhancement with different speeds based on design of experiment method (DOE) method," Journal of Mechanical Engineering Research, vol. 5, no. 3, pp. 50-59, 2013.

[19] P. Mohan, D. Marzougui, E. Arispe, and C. Story, "Component and full-scale tests of the 2007 chevrolet silverado suspension system," NCAC Test Report, The George Washington University, Washington, DC, USA, 2009.

[20] M. Arraigada and M. Partl, "Calculation of displacements of measured accelerations, analysis of two accelerometers and application in road engineering," in Proceedings of the 6th Swiss Transport Research Conference, vol. 1517, Ascona, Switzerland, 2006.

[21] D. C. Montgomery, Design and Analysis of Experiments, John Wiley \& Sons, Hoboken, NJ, USA, 2009.

[22] P. S. Pillai, "Inflation pressure effect on whole tyre hysteresis ratio and radial spring constant," Indian Journal of Engineering and Materials Sciences, vol. 13, no. 2, pp. 110-116, 2006. 


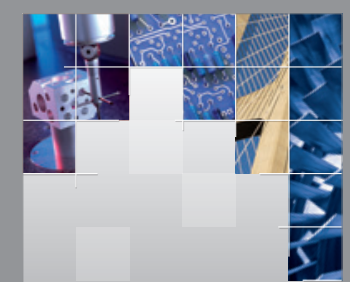

\section{Enfincering}
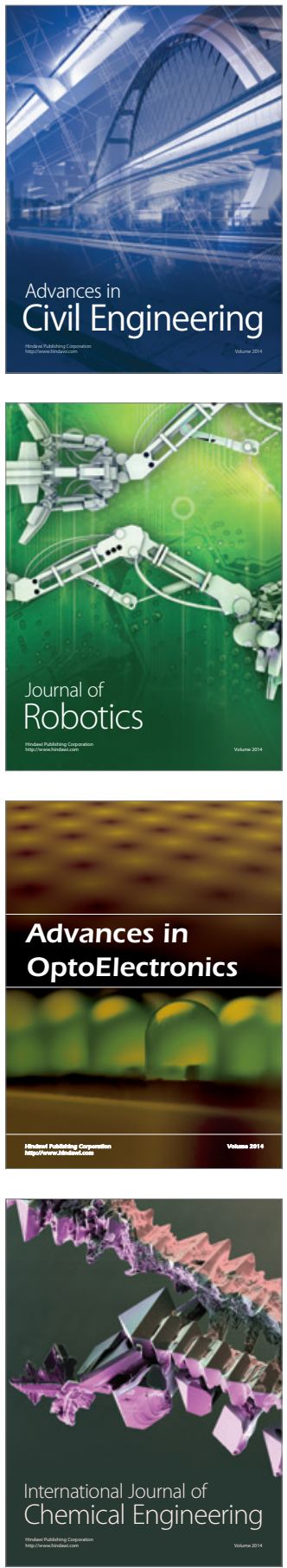

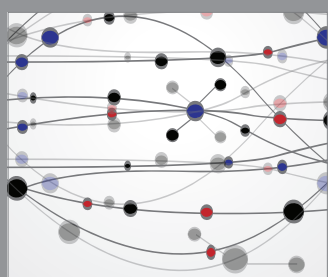

The Scientific World Journal

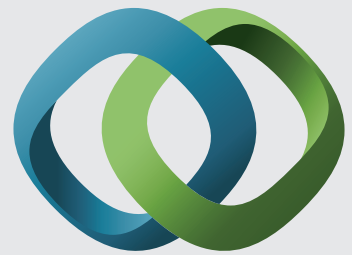

\section{Hindawi}

Submit your manuscripts at

http://www.hindawi.com
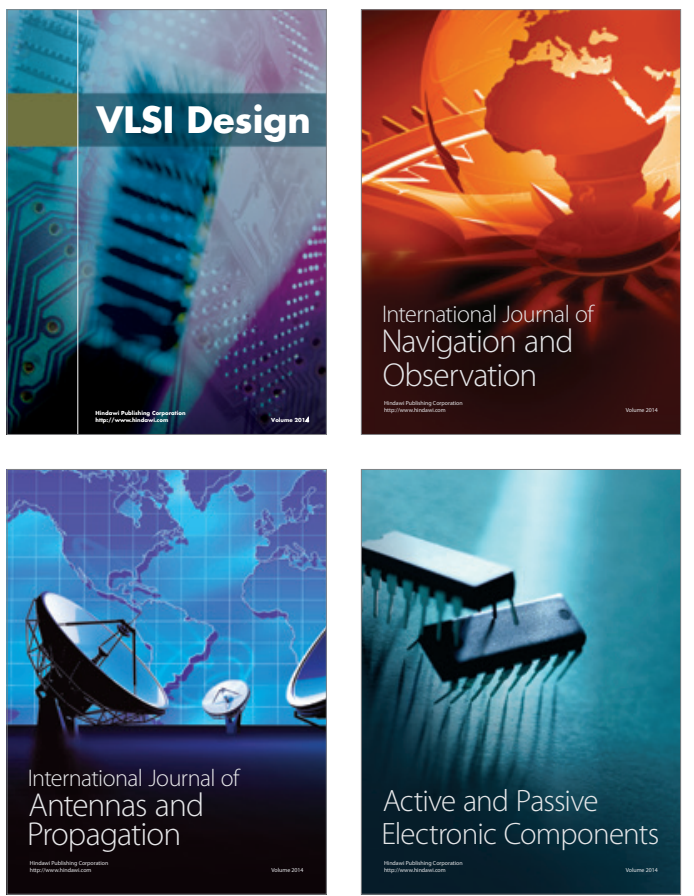
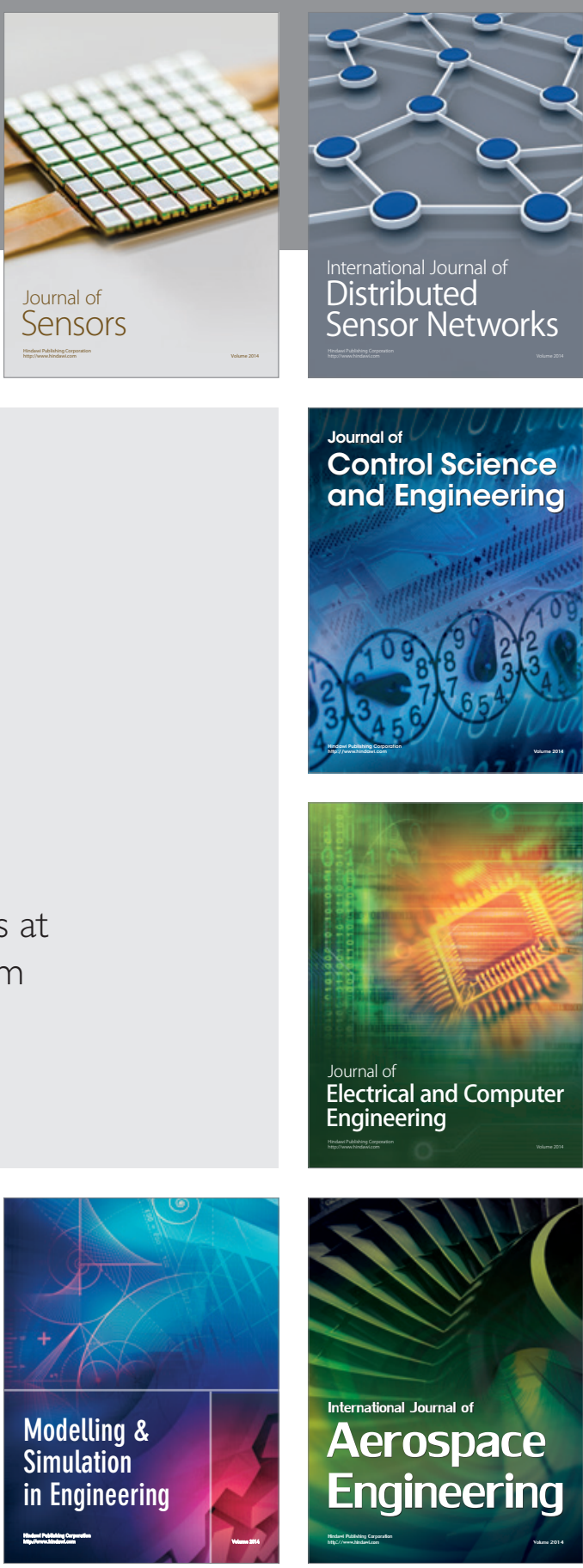

International Journal of

Distributed

Sensor Networks

Journal of

Control Science

and Engineering
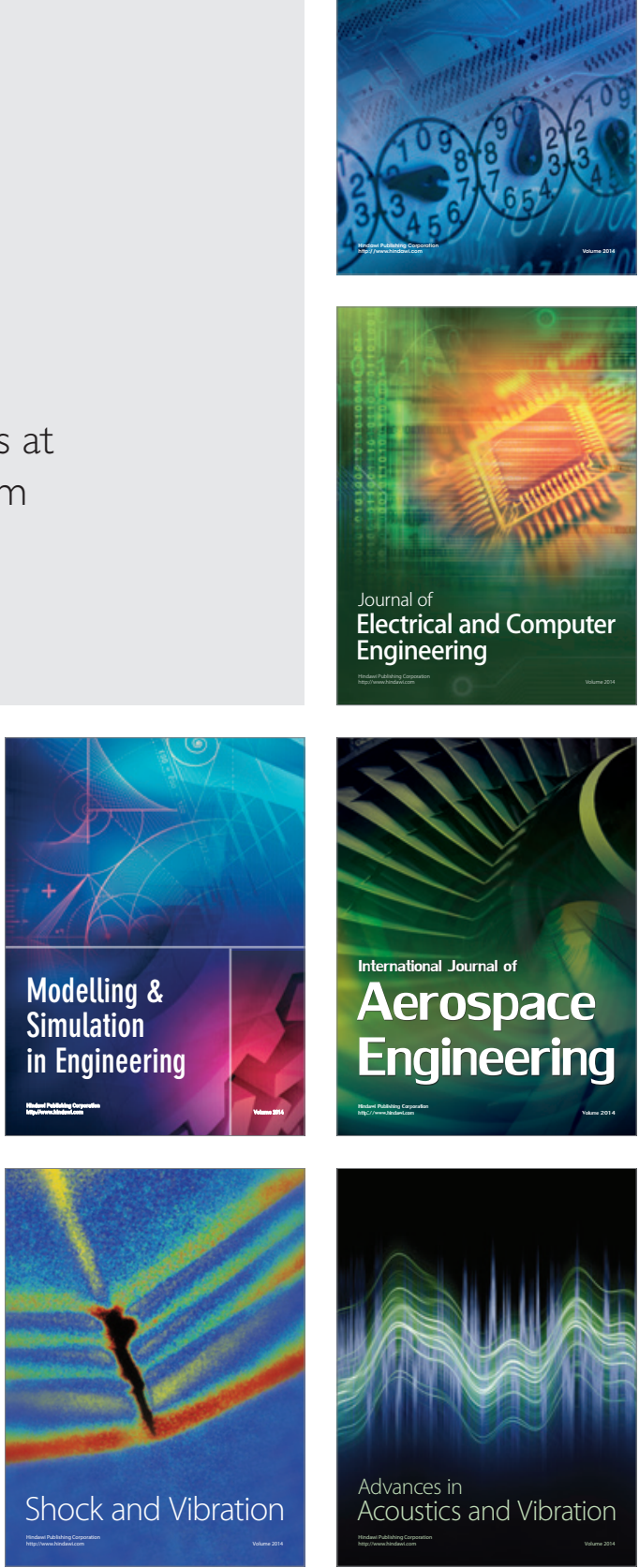